\title{
Analysis of Entry Vehicle Aerothermodynamics Using the Direct Simulation Monte Carlo Method
}

\author{
Jose F. Padilla, ${ }^{*}$ Kun-Chang Tseng, ${ }^{\dagger}$ and Iain D. Boyd ${ }^{\ddagger}$ \\ University of Michigan, Ann Arbor, MI, 48109
}

This paper presents simulations of the aerothermodynamics of the Apollo Command Module under rarefied conditions with the direct simulation Monte Carlo method. Relevant details of the application setup and simulation performance are presented. Threedimensional windtunnel model and flight simulations are made. For the windtunnel model simulations, the direct simulation Monte Carlo method and an analytical free molecular flow method are compared with existing windtunnel data. These simulations are made at different angles-of-attack and Reynolds numbers. The Monte Carlo simulations provide accurate lift and drag results for the windtunnel test. The pitching moment is sensitive to changes in Reynolds number and in center-of-gravity location. Reasonable heat transfer simulation results are obtained. For the flight simulations, the pressure results are reasonable in comparison with the Newtonian theory. The corresponding heat transfer simulation results are reasonable in comparison to available heat transfer empirical data.

\section{Nomenclature}

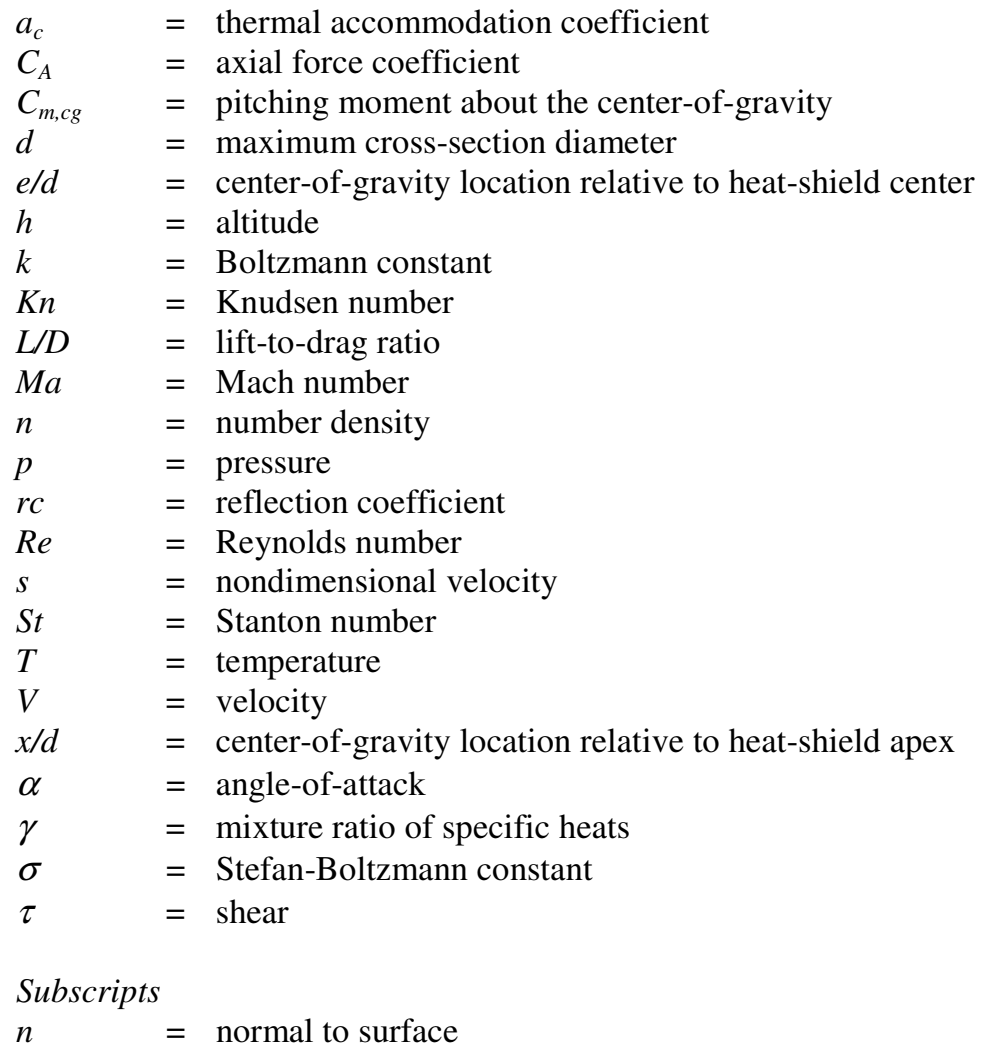

\footnotetext{
* Graduate Student, Department of Aerospace Engineering, Student Member AIAA.

${ }^{\dagger}$ Visiting Graduate Student, Department of Aerospace Engineering.

${ }^{\sharp}$ Professor, Department of Aerospace Engineering, Associate Fellow AIAA.
} 


$\begin{array}{ll}t & =\text { tangent to surface } \\ \text { trim } & =\text { at trim condition } \\ w & =\text { of the wall } \\ 2 \mathrm{~d} & =\text { behind shock wave } \\ \infty & =\text { of the free-stream }\end{array}$

\section{Introduction}

$\mathrm{T}$ HE entering of a planetary atmosphere at a near orbital velocity has been a common scene in the business of space exploration. This scene is expected to become more and more common with the resurgence of manned space exploration. In space exploration, NASA has decided to make it a goal to send people back to the Moon, then to Mars and so forth. Moreover, the rise of the business of space development may have already begun with the recent successful demonstration of a privately funded trans-atmospheric vehicle. However, the process of descending from outer space through an atmosphere is nowhere near perfected. A most notable example was the space shuttle that broke apart in the atmosphere while descending to land less than two years ago. This very sensitive phase of flight must be handled with extreme care. Any subtle failure, as has been observed, could lead to destruction and death. Hence, it is of highest importance to hone the entry phase of space travel to a satisfactory level. In pursuit of this goal, it is necessary to understand the physical phenomena that affect an entry vehicle during the entry phase of flight. In addition, there is expense and danger associated with flight testing and the lessdangerous wind-tunnel testing. These factors are mitigated by computer simulation. This tool cannot eliminate physical experimentation, but it can greatly reduce the amount of such experimentation. There are three major types of vehicles that cross the atmosphere: launch, cruise and entry. Efficient aerodynamics is desirable for launch and, especially, cruise vehicles. The most efficient cruise vehicles have sharp leading edges for minimum shock standoff distance and, thus, maximum lift-to-drag ratio. Contrarily, entry vehicles desire high drag for optimum aerobraking, particularly at the higher altitudes where the atmosphere begins to be felt. There are two major classifications of entry vehicles: those that travel to other planets and moons with atmospheres and those that return to the atmosphere they came from. The former are called, again, entry vehicles, and the latter are called reentry vehicles. Since the analysis of this paper applies to both, the more general classification of entry vehicles is employed. Other than missiles, the only known operational structural configuration of entry vehicles is the blunt or bluff body. There are three major kinds of blunt bodies: winged bodies, lifting bodies and ballistic capsules. The Space Shuttle Orbiter exemplifies the winged body. The X-38, which is a prototype design for an emergency crew-return vehicle for the International Space Station (ISS), provides an example of a lifting body. It has stubby wing-like protrusions in the tail mainly for stability and control. Finally, for the ballistic capsule, there are a handful of examples. In 1961, the first man in space was returned by the Soviet Vostok. Other ballistic capsules of 1960's vintage included the Mercury, Gemini and Apollo. The present expedition crews to the ISS employ the Russian Soyuz. The Crew Exploration Vehicle that will replace the space shuttle is expected to be of this module-capsule type of configuration. There are also various unmanned ballistic capsule entry vehicles. These include the vehicles that successfully entered Mars such as Viking 1 and 2, Pathfinder, Spirit and Opportunity. Another unmanned capsule is the Stardust comet sample return vehicle, which has yet to reenter the Earth's atmosphere, but is currently traveling through space. The analysis of this paper targets ballistic capsule entry vehicles because of their simple geometry, available data and relevance to the present aerospace technology. First, the theory and procedure are described. This includes two numerical methods and two applications of these methods. The results are then presented and discussed. Finally, conclusions are made and possibilities for future work are presented. 


\section{Theory and Procedure}

The general flow regime of entry vehicles at entry altitudes $(\sim 100 \mathrm{~km})$ can be classified as hypersonic, nonequilibrium and dilute. A typical entry velocity from low Earth orbit is Mach 24. Entry velocities from further distances are even larger. This is well into the hypersonic flow regime which generally begins at Mach 5 . There, thermal and chemical flow phenomena become significant. Because of a strong shock wave and high temperatures, thermal and chemical nonequilibrium occur about the vehicle. The associated free-stream Knudsen number decreases from the rarefied regime $\left(>10^{-1}\right)$ to the continuum regime $\left(<10^{-4}\right)$ as the vehicle traverses its descent trajectory. When the local flow is in the rarefied or the upper range of the transitional regime, it is best modeled by a kinetic approach. At entry altitudes, the entire flow field is in this range.

\section{A. Numerical Methods}

\section{Free Molecular Flow Analysis}

When the Knudsen number exceeds 10, collisions between particles become so few that the gas can be considered collisionless. The motion of this gas is called a free molecular flow. For example, properties of rarefied gases experienced in a low Earth orbit above $150 \mathrm{~km}$ for a vehicle with a 1 meter characteristic length can be accurately computed by using free molecular flow analysis. ${ }^{1}$ The properties of interest in entry vehicle analysis are those that affect the vehicle's performance. These include the pressure, shear stress and heat flux. In a free molecular flow, the mean distance between intermolecular collisions is much greater than the characteristic size of the vehicle. The vehicle is assumed to be immersed in an infinite domain of a collisionless gas. Furthermore, because molecules approaching the vehicle surface do not collide with reflected molecules, the free-stream molecules receive no warning about the approaching vehicle until they collide with its surface. Under these conditions expressions for the surface properties can be derived. Consider a flat plate oriented at an angle of attack with respect to the free-stream, fig. 1.

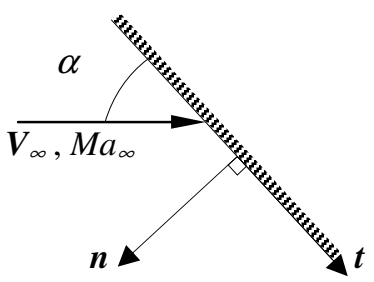

\section{Figure 1. Surface for the free molecular flow analysis}

Now, define a nondimensional velocity according to

$$
s=\sqrt{\frac{\gamma}{2}} M a
$$

It can be shown that the corresponding surface pressure and shear are: ${ }^{1}$

$$
\begin{gathered}
p=p_{\infty}\left\{\left(\frac{2-r c_{n}}{\sqrt{\pi}} s_{n}+\frac{r c_{n}}{2} \sqrt{\frac{T_{w}}{T_{\infty}}}\right)\left(\exp \left(-s_{n}^{2}\right)+\sqrt{\pi} s_{n}\left[1+\operatorname{erf}\left(s_{n}\right)\right]\right)\right\} \\
\tau=p_{\infty} s_{t} r c_{t}\left\{\frac{1}{\sqrt{\pi}} \exp \left(-s_{n}^{2}\right)+s_{n}\left[1+\operatorname{erf}\left(s_{n}\right)\right]\right\}
\end{gathered}
$$

where

$$
p_{\infty}=n_{\infty} k T_{\infty}
$$




$$
s_{n}=-s \sin \alpha
$$

and

$$
s_{t}=s \cos \alpha
$$

These equations are applied to a curved surface by modeling it as a polyhedron of many flat faces. The computational grids employed in the Monte Carlo simulations described below provide polyhedrons comprised of triangular faces for modeling the vehicle surfaces.

\section{Direct Simulation Monte Carlo Method}

To analyze the high altitude flow field, the direct simulation Monte Carlo (DSMC) method ${ }^{2}$ is employed. It is a physically based probabilistic numerical simulation method. It is not a numerical solution to the classical Boltzmann equation, but uses the same underlying physics. Unlike the deterministic molecular dynamics method, it probabilistically selects collision processes for analysis. The procedures used to process these collision processes are based on kinetic theory. Hence, the method is limited to nonequilibrium weakly ionized dilute gases with thermochemical behavior. This, indeed, encompasses all the significant physical behavior of flow about an entry vehicle at an entry altitude.

This study employs the general, object-oriented, cell-based, parallelized implementation of the DSMC method called MONACO. ${ }^{3}$ It uses the Variable Soft Sphere (VSS) collision model, ${ }^{4}$ and variable vibrational ${ }^{5}$ and rotational $^{6}$ energy exchange probability models. Within it, the Total Collision Energy (TCE) model ${ }^{2}$ regulates chemical $^{2}$ reactions. Accommodation coefficients handle collisions between particles and a wall surface. They indicate the fraction of specular and diffuse reflections. MONACO can handle three-dimensional unstructured tetrahedral computational grids with cell weighting factors.

\section{B. Applications}

\section{Windtunnel Simulations}

In May, 1968, results of hypersonic wind-tunnel tests of small scale models of the Apollo Command Module (ACM) were presented by Boylan and Griffith. ${ }^{7}$ The tests were performed at the Arnold Engineering Development Center (AEDC), and involved a low density, hypersonic, continuous-flow, arc heated, ejector-pumped windtunnel called AEDC Tunnel L. Table 1 lists the flow conditions. The angle-of-attack is referenced with respect to the acute side of the vehicle, the side that the pilot faces. Figure 2 illustrates this and the location of the center-of-gravity referenced to the center and apex of the heat-shield. The dimensionless distance $x / d$ is fixed while the perpendicular offset $e / d$ is allowed to vary. $d$ is the maximum cross-section diameter of the vehicle.

Table 1. Reference conditions for the windtunnel model simulation

\begin{tabular}{|l|l|l|}
\hline Property & \multicolumn{2}{|l|}{ Value } \\
\hline Gas & $\mathrm{N}_{2}$ \\
$T_{\infty}, \mathrm{K}$ & 142 & \\
$M a_{\infty}$ & 10.2 \\
$T_{w}, \mathrm{~K}$ & 300 & \\
\cline { 2 - 3 } & Case 1 & Case 2 \\
\cline { 2 - 3 }$e_{\infty}$ & 160 & 230 \\
$K n_{\infty}$ & 0.096 & 0.067 \\
$R e_{2 \mathrm{~d}}$ & 20 & 30 \\
$d, \mathrm{~m}$ & $1.016 \times 10^{-2}$ & $1.524 \times 10^{-2}$ \\
\hline
\end{tabular}

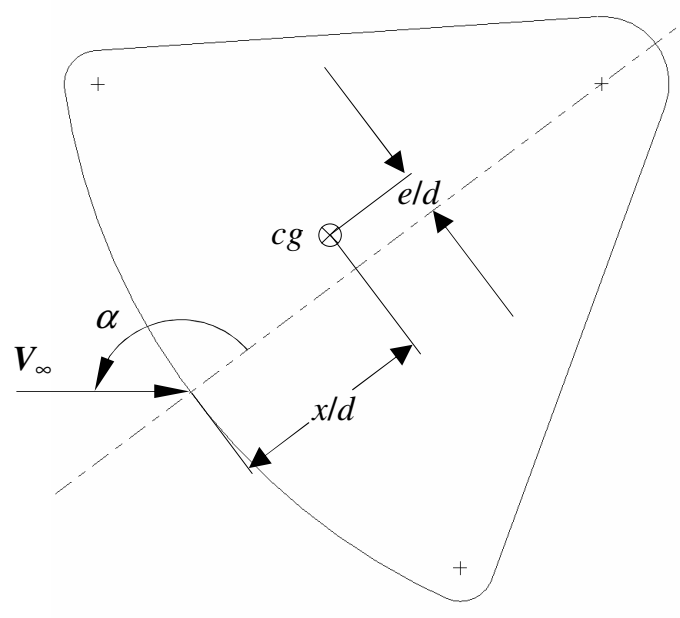

Figure 2. Angle-of-attack and location of center-ofgravity 
Figure 3 defines the computational domain boundaries. They are created with Pro/ENGINEER. ${ }^{8}$ The profile is rotated $180^{\circ}$ to generate the three-dimensional domain boundaries. Because of symmetry about the $x y$ plane, only half of domain is necessary. The region containing only the positive half of the $z$ axis is used. The whole model is then canted to meet the desired angle-of-attack. The grids are generated with HyperMesh ${ }^{9}$ and employ unstructured tetrahedral cells with cell weighting factors. Figure 4 illustrates the computational domain for the $\operatorname{Re}_{2 \mathrm{~d}}=30$ case at a $170^{\circ}$ angle-of-attack. The shock Reynolds number $R_{2 \mathrm{~d}}$ is the Reynolds number of the flow right behind the shock wave. ${ }^{7}$ The windtunnel models had different diameters at different values of $\operatorname{Re}_{2 \mathrm{~d}}$. For the test cases with shock Reynolds numbers of 20 and 30, the models are $0.26 \%$ and $0.39 \%$ the full size of the ACM, respectively. Threedimensional simulations are run for all cases. Table 2 describes a typical simulation performance. Simulations are made at $\alpha=180^{\circ}, 170^{\circ}, 160^{\circ}$, and $150^{\circ}$, each for $\operatorname{Re}_{2 \mathrm{~d}}=20$ and 30 .

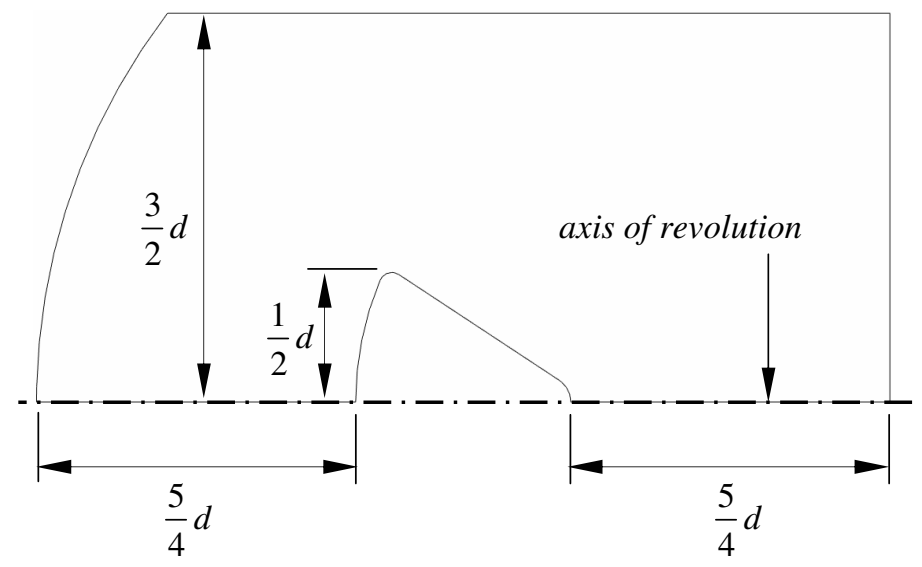

Figure 3. Computational domain boundary profile for windtunnel model simulation, profiles are revolved $180^{\circ}$ about axis of revolution

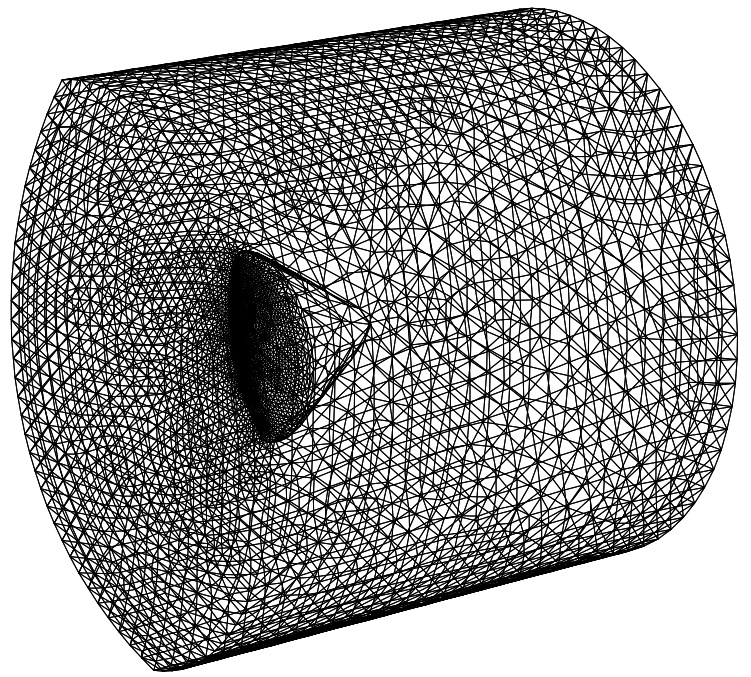

Figure 4. Computational grid boundary, $R e_{2 \mathrm{~d}}=30, \alpha=170^{\circ}$ 
Table 2. Typical windtunnel simulation performance

\begin{tabular}{|l|l|}
\hline Property & Value \\
\hline$R e_{2 \mathrm{~d}}$ & 30 \\
$\alpha$ & $170^{\circ}$ \\
Cells & 125,775 \\
Particles & $8,000,000(\sim 0.4 \%$ uncertainty $)$ \\
Time step & $1.5 \times 10^{-8} \mathrm{~s}$ \\
Number of time steps & $60,000(53,000$ sampled $)$ \\
CPUs & $1.4 \mathrm{GHz} \times 8$ \\
Simulation time & $10 \mathrm{hr}$ and $54 \mathrm{~min}$ \\
\hline
\end{tabular}

\section{Flight Simulations}

In April, 1968, the Apollo 6 mission was conducted. ${ }^{10}$ It involved an unmanned test flight of the ACM with an inertial entry velocity of $10.0 \mathrm{~km} / \mathrm{s}$ into the Earth's atmosphere. Surface pressure and heating rate history data were gathered by using pressure transducers, radiometers and calorimeters. From these results, we choose to simulate the $100 \mathrm{~km}$ point on the entry trajectory because it provided a manageable simulation starting point with some available data for comparison. Table 3 lists the relevant flow conditions and simulation parameters (the simulation times are not available). The MSIS-E-90 atmosphere model ${ }^{11}$ is used to determine the free-stream thermodynamic properties. The wall temperature was set by assuming that the free-stream kinetic energy is fully radiated at the surface:

$$
\frac{1}{2} \rho_{\infty} V_{\infty}^{3}=\sigma T_{w}^{4}
$$

A set of 29 chemical reactions are employed. Figure 5 illustrates the computational domain. Unstructured tetrahedral cells with weighting factors are again used. Simulations are made with two accommodation coefficients, 1.00 and 0.85 .

Table 3. Reference conditions and simulation performance for the flight simulations

\begin{tabular}{|l|l|}
\hline Property & Value \\
\hline$h$ & $100 \mathrm{~km}$ \\
Gas & 5 species air: $\mathrm{N}_{2}, \mathrm{O}_{2}, \mathrm{NO}, \mathrm{N}, \mathrm{O}$ \\
$V_{\infty}$ & $9.6 \mathrm{~km} / \mathrm{s}$ \\
$T_{\infty}$ & $191 \mathrm{~K}$ \\
$n_{N_{2}, \infty}$ & $8.47 \times 10^{18} \mathrm{~m}^{-3}$ \\
$n_{O_{2}, \infty}$ & $2.03 \times 10^{18} \mathrm{~m}^{-3}$ \\
$n_{O, \infty}$ & $4.00 \times 10^{17} \mathrm{~m}^{-3}$ \\
$T_{w}$ & $1378 \mathrm{~K}$ \\
$d$ & $3.91 \mathrm{~m}$ \\
$\alpha$ & $155^{\circ}$ \\
Cells & 750,000 \\
Particles & $\sim 15,000,000$ \\
Time step & $5.0 \times 10^{-7} \mathrm{~s}$ \\
Time steps & $50,000(45,000$ sampled $)$ \\
CPUs & $1.4 \mathrm{GHz} \times 12$ \\
\hline
\end{tabular}




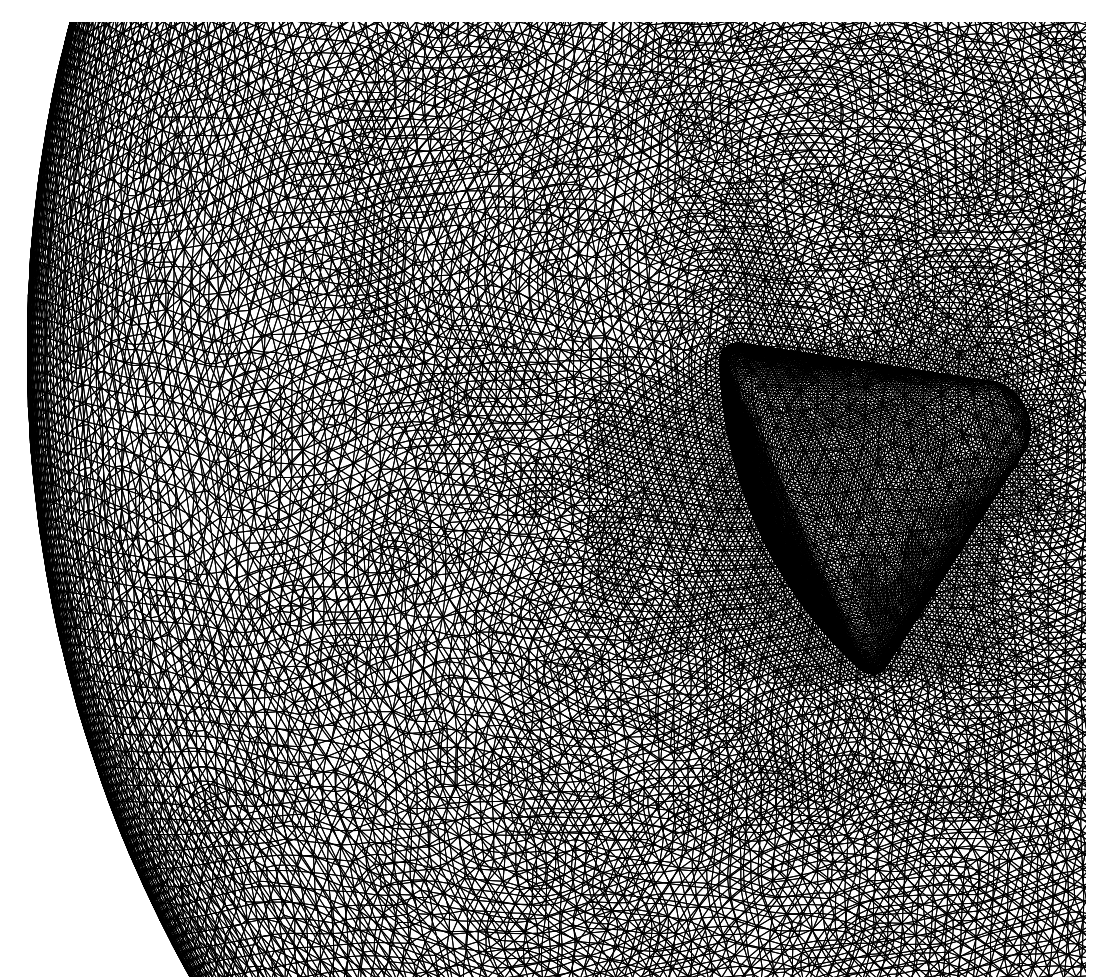

Figure 5. Illustration of computational grid boundary for flight simulation

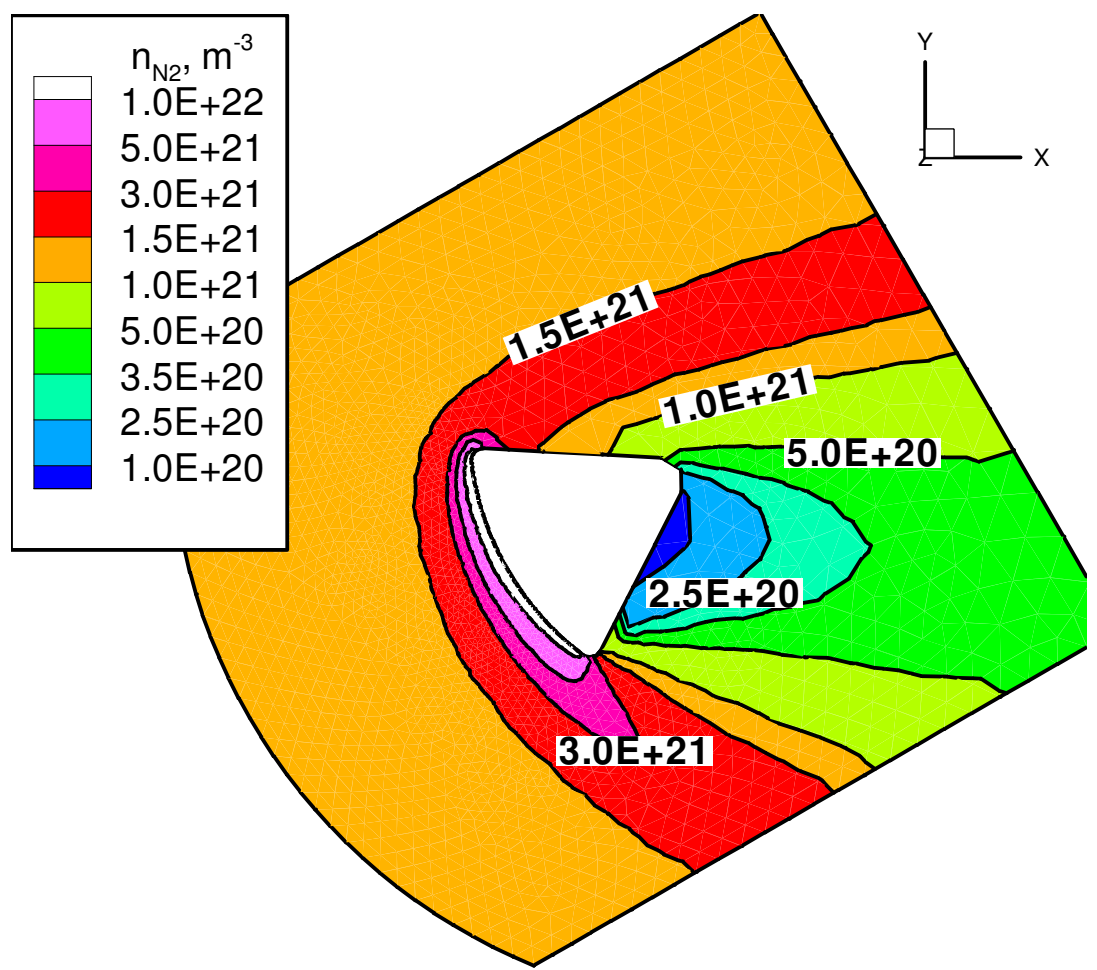

Figure 6. Number density contours in plane of symmetry of windtunnel simulation, $\operatorname{Re}_{2 \mathrm{~d}}=30, \alpha=150^{\circ}$ 


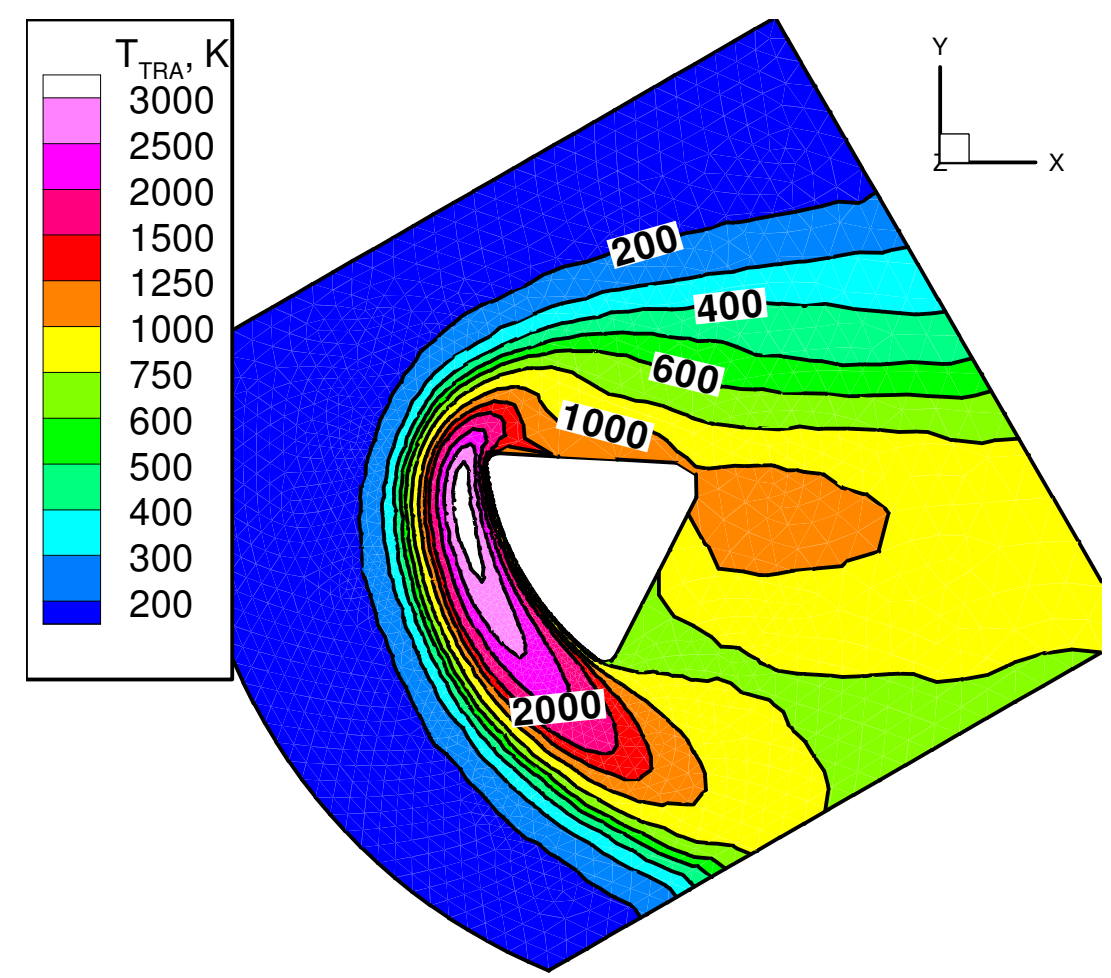

Figure 7. Translational temperature contours in plane of symmetry of windtunnel simulation, $\operatorname{Re}_{2 \mathrm{~d}}=30, \alpha=150^{\circ}$

\section{Results}

\section{A. Windtunnel Simulations}

Figures 6 and 7 are contour plots of density and translational temperature in the plane of symmetry for the case where $\operatorname{Re}_{2 \mathrm{~d}}=30$ and $\alpha=150^{\circ}$. They place the flow field in perspective and provide a general indication of whether the simulation is satisfactory. The computational domain is adequate for capturing the major flow phenomena about the vehicle. The vehicle's presence incurs large gradients which indicate the existence of a bow shock, as expected. The qualities of the compression and expansion regions ahead of and behind the vehicle, respectively, are reasonable. For example, peak densities and temperatures occur near the front most part of the vehicle. The peak temperature $(3260 \mathrm{~K})$ is less than the initial dissociation temperature of nitrogen $(4000 \mathrm{~K})^{12}$, thus the exclusion of chemistry in this simulation is justified. All windtunnel simulations did not require chemistry computations, as expected.

Figures 8 through 12 summarize the aerodynamic results of the windtunnel test simulations. The effect of varying angle-of-attack, shock Reynolds number and center-of-gravity location, and of intermolecular collisions are examined. The figures demonstrate that the free molecular flow analysis given by MONACO is in complete agreement with the corresponding analytical analysis. ${ }^{1}$

Figure 8 shows the variation of lift-to-drag ratio with angle-of-attack and indicates that the DSMC simulations given by MONACO agree well with the windtunnel data. This includes the qualitative trend that lift-to-drag ratio increases with Reynolds number. ${ }^{7}$ As expected, the free molecular flow calculations result in a much smaller increase of lift-to-drag ratio with angle-of-attack.

Figure 9 shows that there is less agreement between MONACO and the available axial force windtunnel data. Nevertheless, the DSMC simulations provide the correct trends and are still within 6\% accuracy. The free molecular flow calculations also provide qualitatively correct trends, however, their accuracy is much worse. As expected, the axial force coefficient increases with shock Reynolds number.

Figure 10 indicates that the windtunnel pitching moment data is more difficult to capture by the simulations. The trends are in agreement with existing literature. The moment curve slope increases with gas rarefaction and even changes sign. ${ }^{13,14}$ Thus, the vehicle's pitch stability goes from being statically unstable to statically stable as it 
descends into the atmosphere. The figure does not consider the effect of changing the accommodation coefficient. This has been shown to significantly affect such results. ${ }^{15}$ Free molecular flow analysis gives results in line with the previously discussed trend. It predicts static pitch instability for rarefied flow. It is inadequate in predicting the vehicle pitch behavior under these flow conditions.

Figures 11 and 12 examine the sensitivity of pitching moment and trim conditions with changes in center-ofgravity location. Figure 11 shows that a decrease in the center-of-gravity vertical offset causes a commensurate shift in the pitching moment curve within the range of angles-of-attack studied. Sensitivity of pitching moment to changes in center-of-gravity location is about the same for the collisional and free molecular flow analyses. Thus, the center-of-gravity location affects the trim angle-of-attack independently of moment slope changes. Figure 12 shows that changing the center-of-gravity vertical offset $e / d$ by roughly $1 \%$ causes the trim angle-of-attack to change by about $10^{\circ}$ and the lift-to-drag ratio to change dramatically. It also shows that gas rarefaction makes these changes even larger. Thus, shock Reynolds number affects static pitch stability and trim angle-of-attack, center-of-gravity location affects trim angle-of-attack, and both affect trim lift-to-drag ratio or trim performance. Linear interpolation between simulation points and linear extrapolation limit the accuracy, but qualitative trends are obtained.

Figure 13 documents the simulation results for maximum Stanton number on the vehicle surface. The increase in shock Reynolds number decreases the maximum Stanton number for both free molecular and collisional flow. This agrees qualitatively with the physics involved in the collisional flow. The increase in shock Reynolds number results in more intermolecular collisions, hence, more gaseous kinetic energy dissipation is imposed on the gas molecules prior to their collision with the vehicle surface. With the free-molecular flow, this logic does not apply. However, the vehicle model's diameter is larger for the larger shock Reynolds number case. It is such that the number density is smaller there. Thus, there are a smaller number of collisions between the free molecular flow and the wall. Hence, the energy flux or heat transfer is smaller there for the larger shock Reynolds number. For these simulations, this aspect must be the key influence in both collisional and free-molecular flow as the shock Reynolds number changes because for both flows the resulting change in maximum Stanton number is similar.

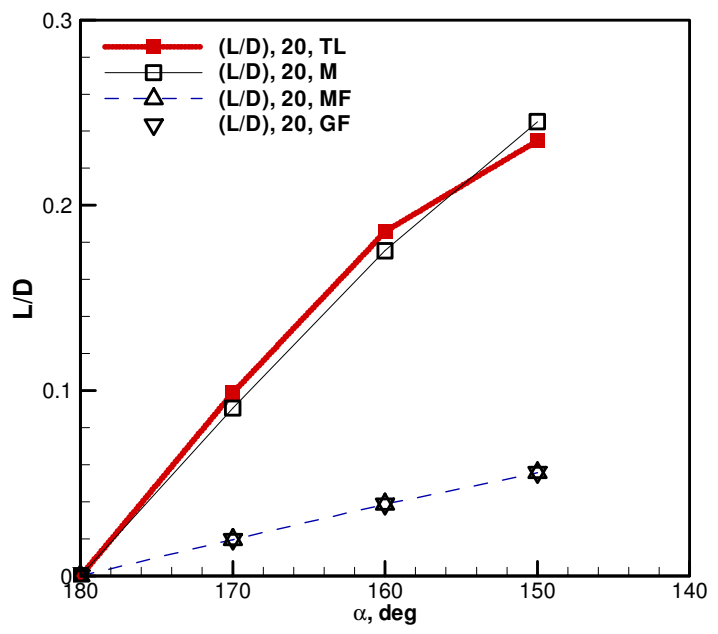

(a) $R e_{2 \mathrm{~d}}=\mathbf{2 0}$

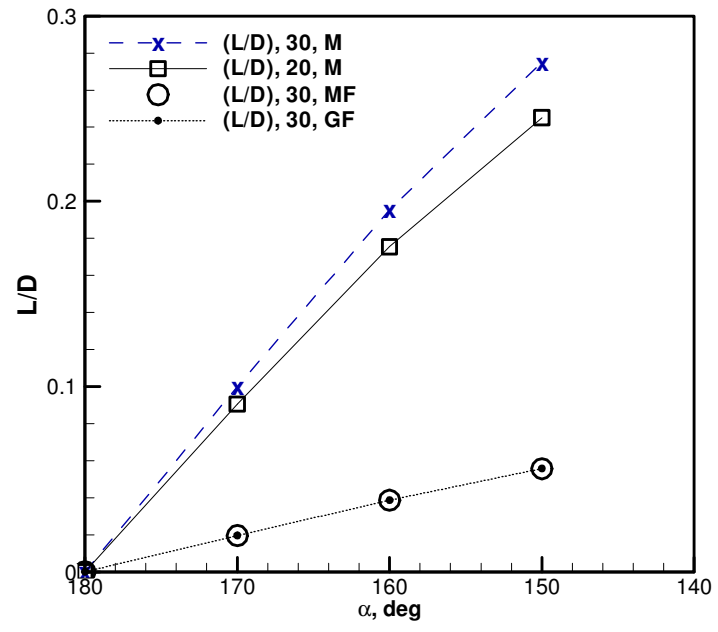

(b) $R e_{2 \mathrm{~d}}=\mathbf{3 0}$

Figure 8. Lift-to-drag as a function of angle-of-attack: $M=M O N A C O, T L=$ windtunnel $L, M F=M$ with free molecular flow, GF=analytical free molecular flow 


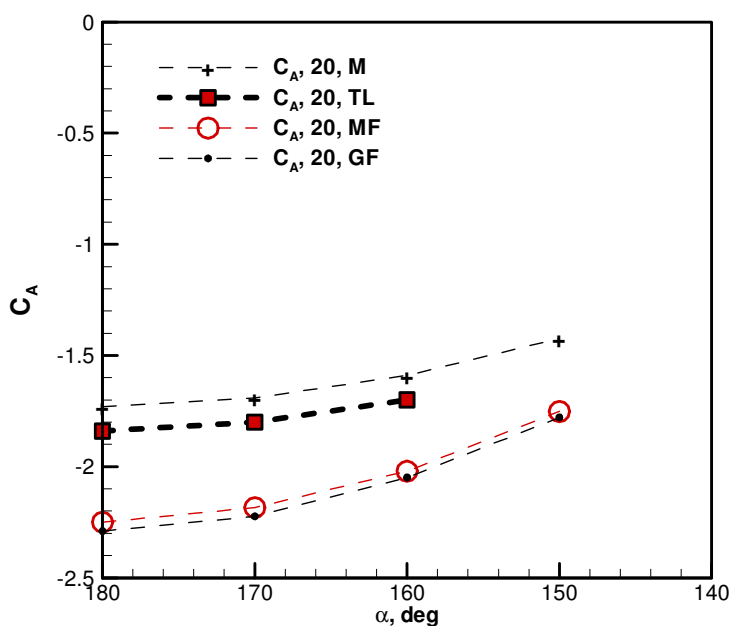

(a) $R e_{2 \mathrm{~d}}=\mathbf{2 0}$

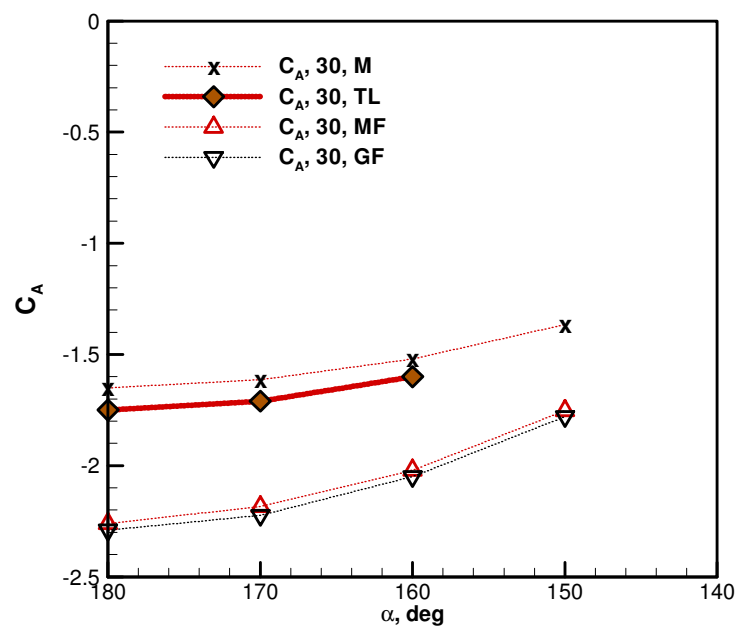

(b) $R e_{2 \mathrm{~d}}=\mathbf{3 0}$

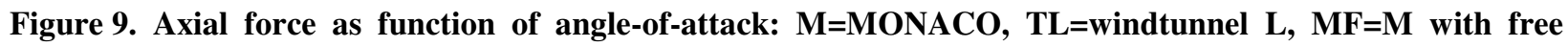
molecular flow, GF=analytical free molecular flow

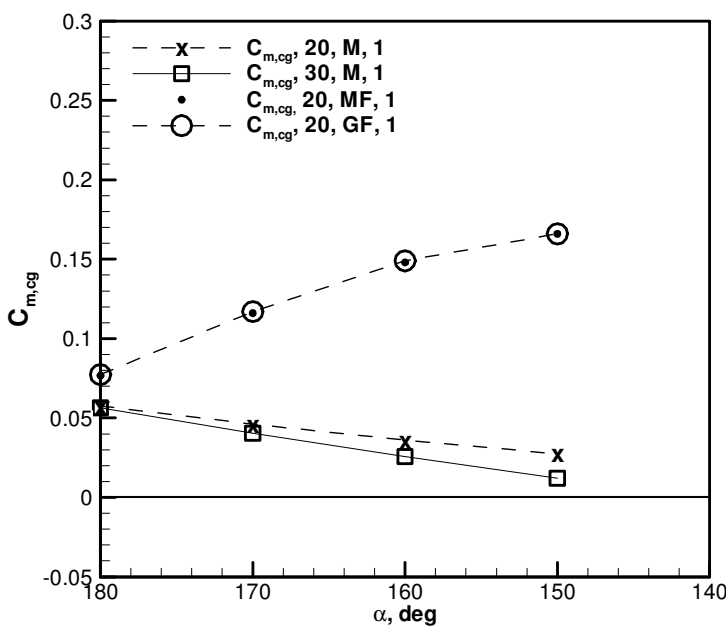

(a) $R e_{2 \mathrm{~d}}=\mathbf{2 0}$

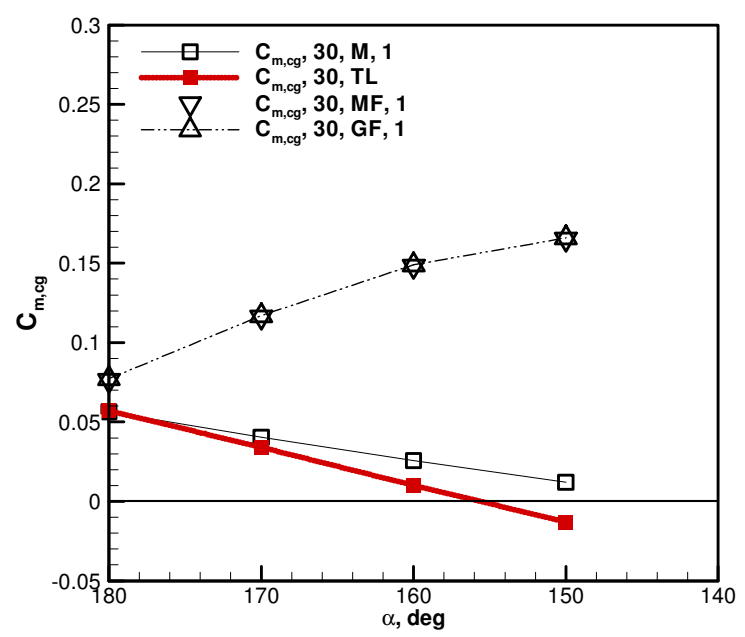

(b) $R e_{2 \mathrm{~d}}=30$

Figure 10. Pitching moment as a function of angle of attack: $M=M O N A C O, T L=$ indtunnel $L, M F=M$ with free molecular flow, $\mathrm{GF}=$ analytical free molecular flow, and $\mathrm{S}=$ sting mounted model 


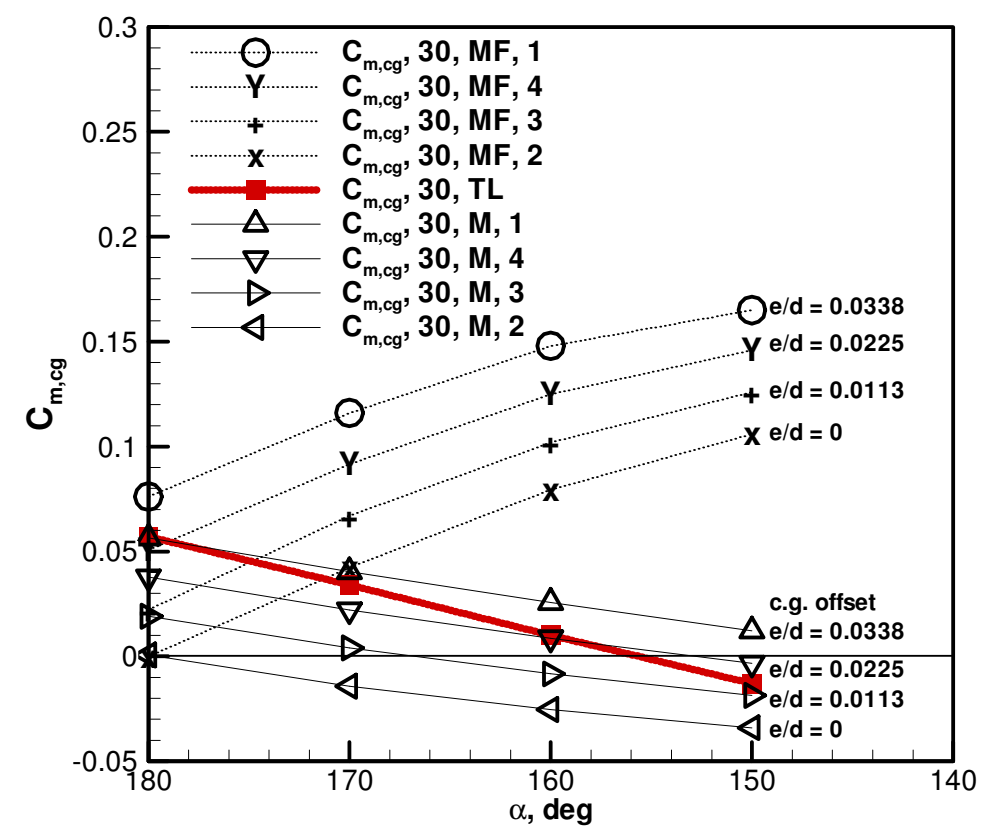

Figure 11. Pitching Moment as a function of angle-of-attack and center-of-gravity location, $R e_{2 \mathrm{~d}}=30$ :, $\mathrm{MF}=\mathrm{M}$ with free molecular flow

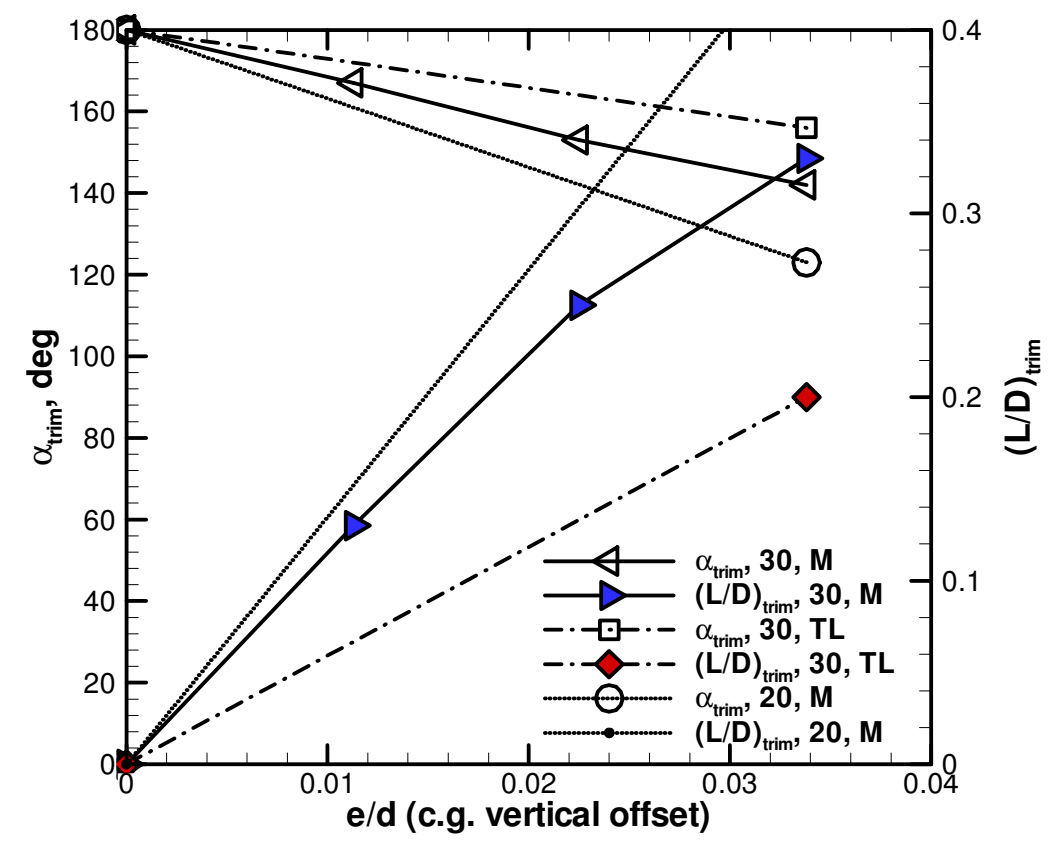

Figure 12. Trim angle-of-attack and lift-to-drag ratio as a function of center-of-gravity vertical offset. $R e_{2 \mathrm{~d}}=20$ and 30. M=MONACO and TL=windtunnel $L$. Linear interpolation and extrapolation was employed. 


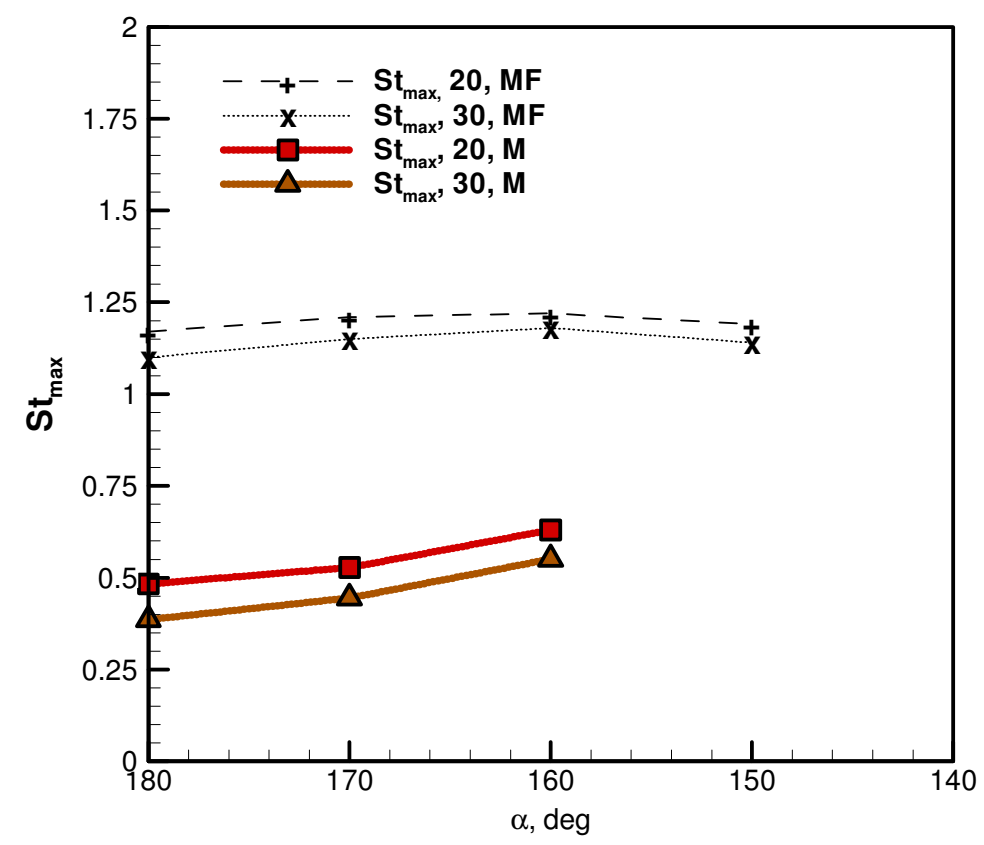

Figure 13. Maximum Stanton Number as a function of angle-ofattack of windtunnel simulation, $R e_{2 \mathrm{~d}}=20$ and 30: M=MONACO, $\mathrm{MF}=\mathrm{M}$ with free molecular flow

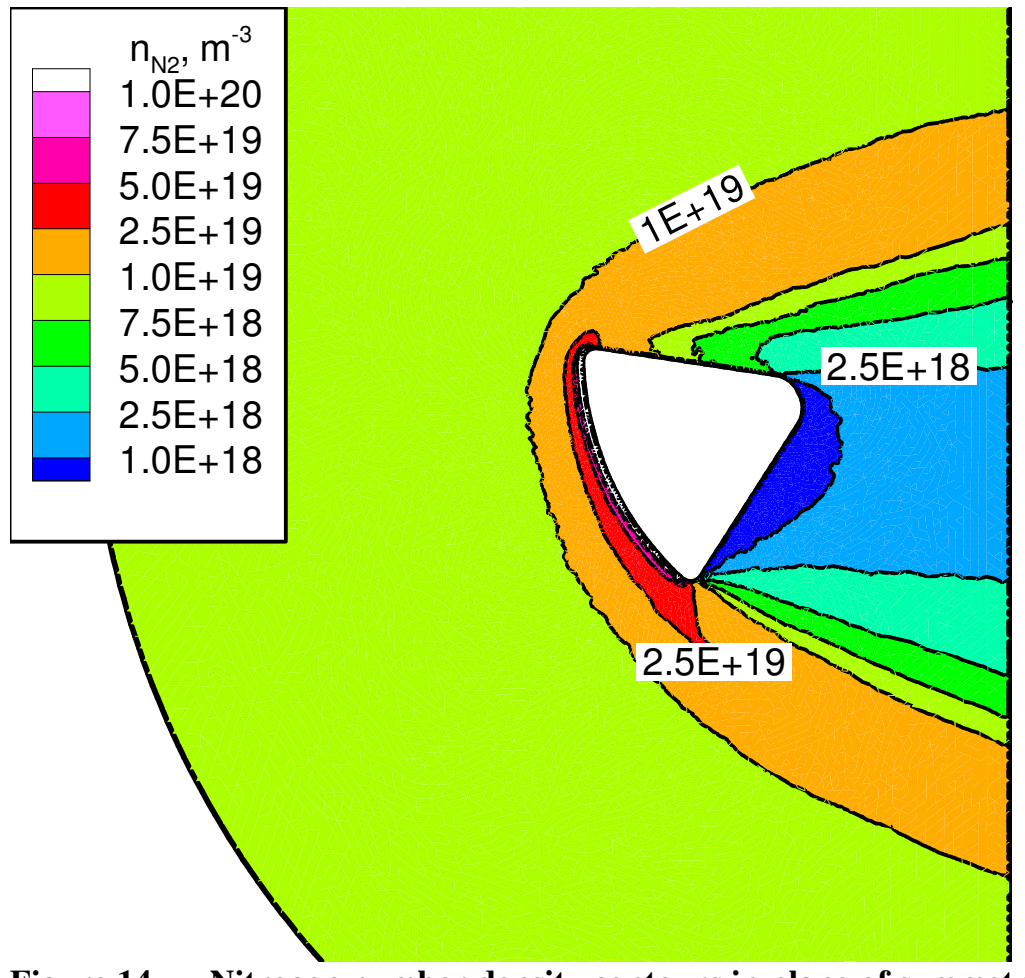

Figure 14. Nitrogen number density contours in plane of symmetry of flight simulation, $\mathrm{h}=100 \mathrm{~km}, \alpha=155^{\circ}, a_{c}=1.00$ 


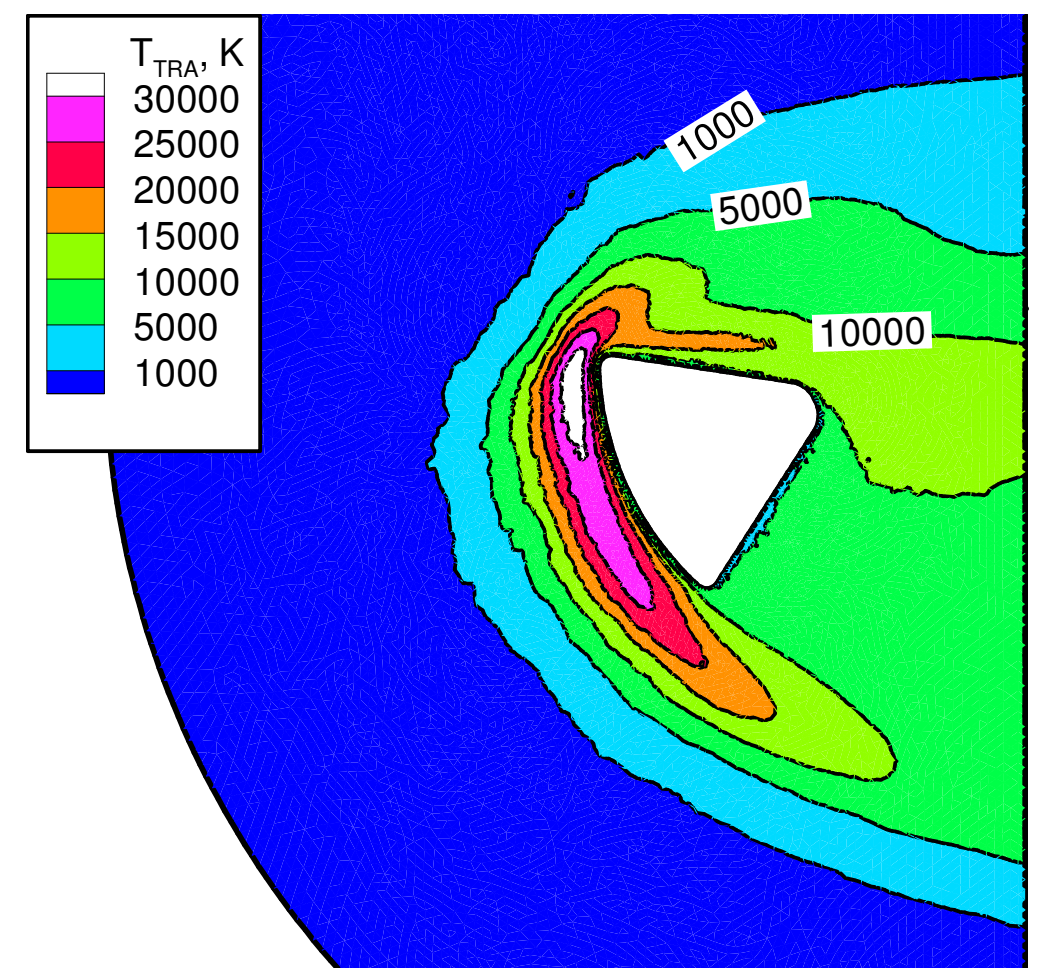

Figure 15. Translational temperature contours in plane of symmetry of flight simulation, $\mathrm{h}=100 \mathrm{~km}, \alpha=155^{\circ}, a_{c}=1.00$

\section{B. Flight Simulation}

Figures 14 and 15 are contour plots of Nitrogen density and translational temperature in the plane of symmetry. As with the windtunnel simulation they are a test of whether the simulation is completed satisfactorily. The computational domain is adequate for capturing the major flow phenomena about the vehicle. The vehicle's presence incurs large gradients which indicate the existence of a bow shock, as expected. The qualities of the compression and expansion regions ahead of and behind the vehicle, respectively, are reasonable. The peak temperature is an order of magnitude greater than in the windtunnel simulation. The flow field is highly chemically active.

Tables 4 and 5 below summarize the aerothermodynamic results of the Apollo 6 Earth entry simulation. Unfortunately, he flight pressure data is highly scattered at $100 \mathrm{~km}$ altitude. ${ }^{16}$ This made it difficult to extract meaningful pressure data. The DSMC pressure results show promise when compared with the Newtonian theory. The simulation is closer to the Newtonian theory when the accommodation coefficient is 0.85 . Qualitative agreement does appear among all pressure data. The pressure coefficient decreases in going from point 1 to point 3 .

Unlike the pressure data, meaningful heat transfer data is reported ${ }^{16}$ and listed in table 5 . In addition, empirical predictions of heat transfer from Ref. 16 are listed in table 5. They place in perspective the results of the DSMC method. As with the empirical predictions, the DSMC method overestimates the flight data, except at point 4 . The DSMC results show best agreement with the flight data at point 4 . Out of the listed points, this is the point furthest from the plane of symmetry. This leads to the conclusion that the three-dimensional aspects of the simulation are correct. However, the heat flux flight data is computed from temperature data which is considerably scattered at 100 $\mathrm{km}$. The qualitative trends of the DSMC results are in agreement with the convective heat flux prediction adjusted for blowing. 
Table 4. Pressure coefficient at selected points on ACM surface at the $100 \mathrm{~km}$ altitude point of the entry trajectory

\begin{tabular}{|l|l|l|l|}
\hline & Point 1 & Point 2 & Point 3 \\
Newtonian Theory & 2.00 & 0.66 & 0.038 \\
$\mathbf{M}, \boldsymbol{a}_{\boldsymbol{c}}=\mathbf{1 . 0 0}$ & 1.63 & 0.57 & 0.140 \\
$\mathbf{M}, \boldsymbol{a}_{\boldsymbol{c}}=\mathbf{0 . 8 5}$ & 1.88 & 0.60 & 0.094 \\
\hline
\end{tabular}

Point 1: $(\mathrm{x}, \mathrm{y}, \mathrm{z})=(0.000,1.948,93.25) \% \mathrm{R}$

Point 2: $(\mathrm{x}, \mathrm{y}, \mathrm{z})=(23.64,-99.75,69.74) \% \mathrm{R}$

Point 3: $(\mathrm{x}, \mathrm{y}, \mathrm{z})=(34.42,-1.740,99.99) \% \mathrm{R}$

Coordinates are relative to intersection between axis of revolution and heat shield surface. $\mathrm{R}$ is the maximum cross-section radius of the ACM.

$\mathrm{M}, a_{c}$ : MONACO DSMC simulation with specified accommodation coefficient

Table 5. Heat Flux $\left(\mathrm{Btu} /\left(\mathrm{ft}^{2} \cdot \mathrm{s}\right)\right)$ at selected points on $\mathrm{ACM}$ surface at the $100 \mathrm{~km}$ altitude point of the entry trajectory

\begin{tabular}{|l|l|l|l|l|}
\hline & Point 1 & Point 2 & Point 3 & Point 4 \\
Flight Data & 2.0 & 2.3 & 1.0 & 4.0 \\
Prediction 1 & 5.0 & 10.0 & 11.0 & 6.0 \\
Prediction 2 & 9.0 & 6.0 & 5.0 & 6.0 \\
$\mathbf{M}, \boldsymbol{a}_{c}=\mathbf{1 . 0}$ & 4.8 & 6.1 & 8.5 & 3.4 \\
$\mathbf{M}, \boldsymbol{a}_{\boldsymbol{c}}=\mathbf{0 . 8 5}$ & 4.7 & 5.8 & 8.5 & 3.2 \\
\hline
\end{tabular}

Point 1: $(\mathrm{s}, \mathrm{y}, \mathrm{z})=(0.400,-0.623,0.623) \% \mathrm{R}$

Point 2: $(\mathrm{s}, \mathrm{y}, \mathrm{z})=(51.20,0.000,50.65) \% \mathrm{R}$

Point 3: $(\mathrm{s}, \mathrm{y}, \mathrm{z})=(103.8,1.701,97.38) \% \mathrm{R}$

Point 4: $(\mathrm{s}, \mathrm{y}, \mathrm{z})=(67.10,16.53,-64.25) \% \mathrm{R}$

Coordinates are relative to intersection between axis of revolution and heat shield surface. $\mathrm{R}$ is the maximum cross-section radius of the ACM. $s$ is the arc-length of the surface between the sensor and the origin of the coordinate system.

Prediction 1: Convective adjusted for blowing plus radiative predictions ${ }^{15}$

Prediction 2: Laminar cold wall plus radiative predictions ${ }^{15}$

$\mathrm{M}, a_{c}$ : MONACO DSMC simulation with specified accommodation coefficient

\section{Conclusion}

This paper presented an effort to simulate the aerothermodynamics of the ACM at high altitudes with the DSMC method. ${ }^{2}$ Relevant details of the application setup and simulation performance were presented. Three-dimensional windtunnel model and flight simulations were made.

For the windtunnel model simulations, results from the DSMC method and an analytical free molecular flow method $^{1}$ were compared with existing windtunnel data. ${ }^{7}$ These simulations were made at different angles-of-attack and Reynolds numbers. The DSMC method provided accurate lift and drag results for the Apollo windtunnel test. The analytical free molecular flow analysis was in agreement with the DSMC method set without collisions. The pitching moment was sensitive to changes in Reynolds number and in center-of-gravity location. Reasonable heat transfer results were obtained.

For the flight simulations, the DSMC method was compared with Newtonian theory, existing flight data ${ }^{16}$ and empirical predictions. ${ }^{16}$ These simulations were made at different values of surface accommodation coefficient. Available pressure data was too scattered, but the pressure results of the DSMC method were reasonable in comparison with the Newtonian theory. The agreement here was generally better when the thermal accommodation was set to $85 \%$. Available heat transfer data was overestimated by the empirical predictions. It was also overestimated by the DSMC method except, ironically, at the point furthest from the plane of symmetry, from the set of points considered. However, the temperature data from which the heat transfer flight data was computed was 
highly scattered at the $100 \mathrm{~km}$ altitude point of the entry trajectory. Thus, the accuracy of the simulation could not be determined from the available data at the $100 \mathrm{~km}$ altitude.

These simulation studies could be improved in several ways. Future studies of the pitching moment should include larger angles-of-attack and the effect of varying accommodation coefficient. Simulations of the Apollo 6 entry trajectory should involve altitudes lower than $100 \mathrm{~km}$. At lower altitudes the simulations will be more computationally expensive. Three-dimensional adapted grids were not employed and should be tried. The parallel domain decomposition procedure should be improved. These two propositions will help improve the accuracy and efficiency of the simulations. Future entry vehicle studies could involve alternative heat transfer simulation models and vehicle configurations. This could include a vehicle shape optimization study. These studies could involve extraterrestrial atmospheres and the effects of ionization.

\section{Acknowledgments}

This work is sponsored by the Space Vehicle Technology Institute, under NASA grant NCC-3989 with joint sponsorship from the Department of Defense.

\section{References}

${ }^{1}$ Gombosi, T. I., Gaskinetic Theory, Cambridge University Press, Cambridge, England, 1994.

${ }^{2}$ Bird, G. A., Molecular Gas Dynamics and the Direct Simulation of Gas Flows, Oxford University Press, 1994.

${ }^{3}$ Dietrich, S., and Boyd, I. D., "Scalar and Parallel Optimized Implementation of the Direct Simulation Monte Carlo Method," Journal of Computational Physics, Vol. 126, 1996, pp. 328-342.

${ }^{4}$ Koura, K., and Matsumoto, H., "Variable Soft Sphere Molecular Model for Air Species," Physics of Fluids, Vol. 4, 1992, pp. 1083-1085.

${ }^{5}$ Vijayakumar, P., Sun, Q., and Boyd, I. D., "Detailed Models of Vibrational-Translational Energy Exchange for the Direct Simulation Monte Carlo Method," Physics of Fluids, Vol. 11, 1999, pp. 2117-2126.

${ }^{6}$ Boyd, I. D., "Analysis of Rotational Nonequilibrium in Standing Shock Waves of Nitrogen," AIAA Journal, Vol. 28, 1990, pp. 1997-1999.

${ }^{7}$ Boylan, D. E., and Griffith, B. J., "Simulation of the Apollo Command Module Aerodynamics at Re-entry Altitudes," Proceedings of the $3^{\text {rd }}$ National Conference on Aerospace Meteorology, American Meteorological Society, Boston, 1968, pp. 370-378.

${ }^{8}$ Pro/ENGINEER, Wildfire, Parametric Technology Coorporation, Needham, MA, 2002.

${ }^{9}$ HyperMesh, Version 3.0, Altair Engineering Inc., Troy, MI, 1999.

${ }^{10}$ Griffith, B. J., and Boylan, D. E., "Reynolds and Mach Number Simulation of Apollo and Gemini Re-entry and Comparison with Flight," Specialists' Meeting on Hypersonic Boundary Layers and Flow Fields of the Fluid Dynamics Panel of AGARD, North Atlantic Treaty Organization, Paris, 1968, pp. 8-1-8-21.

${ }^{11}$ E. Hedin, "Extension of the MSIS Thermospheric Model into the Middle and Lower Atmosphere," Journal of Geophysical Research, Vol. 96, 1991, p. 1159.

${ }^{12}$ Anderson, J. D., Hypersonic and High Temperature Gas Dynamics, McGraw-Hill Book Company, New York, 1989.

${ }^{13}$ Moss, J. N., Wilmoth, R. G., and Price, J. M., "DSMC Simulations of Blunt Body Flows for Mars Entries: Mars Pathfinder and Mars Microprobe Capsules," AIAA Paper 97-2508, June 1997.

${ }^{14}$ Potter, J. L., and Peterson, S. W., "Local and Overall Aerodynamic Coefficients for Bodies in Hypersonic, Rarefied Flow," $29^{\text {th }}$ Aerospace Sciences Meeting, AIAA, Washington, DC, 1991.

${ }^{15}$ Kazuhisa, F., Inatani, Y., and Hiraki, K., "Attitude Stability of Blunt-Body Capsules in Hypersonic Rarefied Regime," Journal of Spacecraft and Rockets, Vol. 41, No. 6, 2004, pp. 925-931.

${ }^{16}$ Lee, D. B., and Goodrich, W. D., "The Aerothermodynamic Environment of the Apollo Command Module During Superorbital Entry,” NASA TN D-6792, 1972. 